\title{
AN IMPLICIT, NUMERICAL METHOD FOR SOLVING TWO-DIMENSIONAL TIME-DEPENDENT DIFFUSION PROBLEMS*
}

\author{
BY \\ THOMAS A. OLIPHANT \\ Los Alamos Scientific Laboratory, University of California \\ Los Alamos, New Mexico
}

1. Introduction. We develop an implicit scheme for the numerical solution of two-dimensional, time-dependent diffusion problems and other related problems. The underlying principle is the solution of a general set of linear, simultaneous equations which may occur in nine-point differencing schemes.

We apply the method to both linear and non-linear problems. Each time step is solved by an iterative procedure and a convergence condition is derived for the linear case.

The stability of any time-dependent treatment is investigated in terms of its particular differencing scheme independently of our method of solving the simultaneous equations. Our scheme is quite useful when applied to simple, unconditionally stable differencing schemes. Schemes involving more than nine points can be constructed and the method is in principle generalizable to three dimensions.

2. Derivation of the method. The basic partial differential equation including the linear and non-linear cases which we wish to consider is

$$
\nabla \cdot\left\{f(\mathbf{r}, \theta) \nabla\left(\theta^{p}\right)\right\}+\lambda(\mathbf{r}, \theta) \theta-S(\mathbf{r})=K \frac{\partial \theta}{\partial t} .
$$

This is not the most general type of equation to which this method is applicable, but it illustrates the generality of the method. We are specifically interested here in approximating the solution to this sort of equation by the use of finite differencing schemes. Generally speaking, implicit schemes are written in the form of a set of linear or nonlinear simultaneous equations for the dependent variables over the mesh at an advanced time. Various implicit methods of this type have proved to be of considerable practical use. One such method is the Douglas-Peaceman alternating direction method [1]. It represents the application of the one-dimensional Bruce, Peaceman, Rachford, and Rice method [2] to two dimensions. In this method one direction is treated exactly at each time step. An alternative method constructed by Baker and Oliphant [3] permits an exact treatment over the entire two-dimensional mesh for a single time step. However, this method has a practical limitation in the symmetry which must be imposed on the second partial derivative term in order that the factorization which is involved can be carried out. The method which we propose here does not have this limitation. However, as we shall see, this method is intimately related to the Baker-Oliphant method.

We wish to approximate the partial differential equation (1) by a finite differencing scheme. We write our differencing scheme as a set of simultaneous equations

$$
B_{k i}^{i j} \theta_{i j}^{n}=D_{k l} \text {. }
$$

Unless otherwise specified, we sum over repeated indices. The $(k, l)$ and $(i, j)$ indices

*Received October 11, 1960; revised manuscript received January 23, 1961. Work done under the auspices of the Atomic Energy Commission. 
refer to the position in two-dimensional space of the mesh points and the $n$ index refers to the advanced time. The $D_{k 1}$ contains such things as source terms and $\theta_{i j}^{n-1}$ and $\theta_{i j}^{n-2}$, the values of $\theta_{i j}$ at earlier times. In non-linear problems, the coefficients $B_{k l}^{i i}$ and $D_{k l}$ may also depend on the $\theta_{i j}^{n}$. In order to avoid confusion in the rest of this section, we will leave the advanced time index $n$ understood. We will write out specific differencing schemes in Sec. 5. The present method is a straightforward generalization of the Bruce, Peaceman, Rachford, and Rice method to two dimensions. We do not factor the quantity $B_{k l}^{i i}\left(B_{k l}^{i j}=A_{k}^{i} B_{l}^{i}\right)$ as was done by Baker and Oliphant. However, we must still use a nine-point differencing scheme rather than a five-point one in order to make the equations all consistent with each other. Therefore, in Eq. (2), $i$ and $j$ differ from $k$ and $l$, respectively, by as much as, but no more than, unity.

The first step in the solution of (2) is to triangulate the quantity $B_{k l}^{i j}$, i.e., to write it as

$$
B_{k l}^{i j}=w_{k l}^{m n} b_{m n}^{i j},
$$

where $w_{k l}^{m n}$ has all zero elements except when

$$
\begin{aligned}
m & =k, k-1 \\
n & =l, l-1
\end{aligned}
$$

and $b_{m n}^{i j}$ has all zero elements except when

$$
\begin{aligned}
& i=m, m+1 \\
& j=n, n+1 .
\end{aligned}
$$

Just as in the one-dimensional case, we set the diagonal element $b_{k l}^{k l}$ (no sum) equal to unity (although other normalizations could be used). Next, we write $D_{k l}$ as

$$
D_{k l}=w_{k l}^{m n} g_{m n} .
$$

Substituting (3) and (6) into (2), we get

$$
w_{k l}^{m n} b_{m n}^{i i} \theta_{i j}=w_{k l}^{m n} g_{m n},
$$

so that we must have

$$
g_{m n}=b_{m n}^{i i} \theta_{i j} .
$$

Equation (3) can now be written out in algebraic form as a set of equations with no sums over indices

$$
\begin{aligned}
w_{i i}^{i-1 i-1} & =B_{i j}^{i-1 i-1} \\
w_{i j}^{i-1 i} & =B_{i i}^{i-1 i}-w_{i j}^{i-1 i-1} b_{i-1 j-1}^{i-1 j} ; \\
w_{i j}^{i j-1} & =B_{i j}^{i j-1}-w_{i j}^{i-1 i-1} b_{i-1 j-1}^{i j-1} ; \\
w_{i j}^{i j} & =B_{i j}^{i j}-w_{i j}^{i-1 i-1} b_{i-1 i-1}^{i j}-w_{i j}^{i-1 j} b_{i-1 j}^{i j}-w_{i j}^{i j-1} b_{i j-1}^{i j} ; \\
b_{i j}^{i+1 i+1} & =\frac{B_{i j}^{i+1 i+1}}{w_{i j}^{i j}} \\
b_{i j}^{i+1 j} & =\frac{B_{i j}^{i+1 j}-w_{i j}^{i j-1} b_{i j-1}^{i+1 j}}{w_{i j}^{i j}}
\end{aligned}
$$




$$
\begin{aligned}
b_{i j}^{i j+1} & =\frac{B_{i j}^{i j+1}-w_{i j}^{i-1 i} b_{i-1 j}^{i j+1}}{w_{i j}^{i j}} \\
B_{i j}^{i-1 i+1} & =w_{i j}^{i-1 i} b_{i-1 j}^{i-1 i+1} \\
B_{i j}^{i+1 i-1} & =w_{i j}^{i j-1} b_{i j-1}^{i+1 i-1} .
\end{aligned}
$$

Equations (9) to (12) serve to determine $w_{i j}^{i-1 i-1}, w_{i j}^{i-1 i}, w_{i j}^{i j-1}$ and $w_{i j}^{i j}$. Equations (13) to (15) serve to determine $b_{i j}^{i+1 i+1}, b_{i j}^{i+1 i}$, and $b_{i j}^{i i+1}$. Unfortunately, however, Eqs. (16) and (17) involve only quantities which have already been determined by calculations at previous mesh points, and in general they are not satisfied as identities. This inconsistency can be removed if we go to an iterative scheme. To do this we write out Eq. (2) and add and subtract quantities from the left side. The resulting equation (18) is so far exactly equivalent to the original equation (2).

$$
\begin{aligned}
B_{i j}^{i-1 i-1} \theta_{i-1 j-1}+B_{i j}^{i-1 j} \theta_{i-1 i} & +\left(B_{i j}^{i-1 i+1}-\beta_{i j}^{i-1 j+1}+\beta_{i j}^{i-1 j+1}\right) \theta_{i-1 i+1}+B_{i j}^{i j-1} \theta_{i j-1} \\
& +B_{i j}^{i j} \theta_{i j}+B_{i j}^{i j+1} \theta_{i j+1}+\left(B_{i j}^{i+1 i-1}-\beta_{i j}^{i+1 i-1}\right. \\
& \left.+\beta_{i j}^{i+1 i-1}\right) \theta_{i+1 i-1}+B_{i j}^{i+1 i} \theta_{i+1 j}+B_{i j}^{i+1 i+1} \theta_{i+1 i+1}=D_{i j} .
\end{aligned}
$$

Transposing appropriate terms to the right hand side, we get

$$
\begin{array}{r}
B_{i j}^{i-1 i-1} \theta_{i-1 i-1}+B_{i j}^{i-1 j} \theta_{i-1 j}+\beta_{i j}^{i-1 j+1} \theta_{i-1 i+1}+B_{i j}^{i j-1} \theta_{i j-1}+B_{i j}^{i j} \theta_{i j}+B_{i j}^{i j+1} \theta_{i j+1} \\
+\beta_{i j}^{i+1 i-1} \theta_{i+1 j-1}+B_{i j}^{i+1 j} \theta_{i+1 i}+B_{i j}^{i+1 i+1} \theta_{i+1 i+1}=E_{i j},
\end{array}
$$

where

$$
E_{i j}=D_{i j}-\left\{\left(B_{i j}^{i-1 i+1}-\beta_{i j}^{i-1 i+1}\right) \theta_{i-1 i+1}+\left(B_{i j}^{i+1 i-1}-\beta_{i j}^{i+1 i-1}\right) \theta_{i+1 i-1}\right\} .
$$

Now, Eqs. (9) to (15) are as before, but instead of (16) and (17) we have

$$
\begin{aligned}
& \beta_{i j}^{i-1 i+1}=w_{i j}^{i-1 i} b_{i-1 j}^{i-1 j+1}, \\
& \beta_{i j}^{i+1 i-1}=w_{i j}^{i j-1} b_{i j-1}^{i+1 i-1} .
\end{aligned}
$$

These equations determine $\beta_{i j}^{i-1 i+1}$ and $\beta_{i j}^{i+1 i-1}$.

Then, we solve a single time step for a linear problem in the following way. The values of $\theta$ appearing on the right hand side of (19) are now taken to be guessed values to be used in the iterations. After computing the $\beta$ 's and guessing the $\theta^{\prime} \mathrm{s}, E_{i j}$ of (20) is determined. In Eq. (6), replacing $D_{i j}$ by $E_{i j}$, we obtain

$$
g_{i j}=\frac{E_{i j}-w_{i j}^{i j-1} g_{i j-1}-w_{i j}^{i-1 i} g_{i-1 j}-w_{i j}^{i-1 i-1} g_{i-1 j-1}}{w_{i j}^{i j}}
$$

With $E_{i j}$ known, we can compute $g_{i j}$ throughout the mesh. Then, with the $b$ 's and $g$ 's known throughout the mesh, we can compute the $\theta$ 's by Eq. (8) which is written out as follows:

$$
\theta_{i j}=g_{i j}-b_{i j}^{i j+1} \theta_{i j+1}-b_{i j}^{i+1 i} \theta_{i+1 i}-b_{i j}^{i+1 j+1} \theta_{i+1 i+1} .
$$

The values of $\theta_{i j}$ determined in this way can be used to find new guessed values. We will discuss the convergence of the iterative scheme for linear problems in Sec. 4 .

It is important to notice here that the $B_{k l}^{i j}$ are restricted only in forming a ninepoint scheme, but are otherwise completely general. Therefore, there is considerable flexibility in writing appropriate differencing schemes. 
3. The boundary conditions. Very general boundary conditions can be set up. We can set up our formulas to take care of such a region as illustrated in Fig. 1 in the following way. First we define the quantities $\Gamma_{i j}$ and $\Delta_{i i}$.

$$
\begin{aligned}
& \Gamma_{i j}=\left\{\begin{array}{lll}
1 & \text { if } & i j \text { is an internal point } \\
0 & \text { if } & i j \text { is not an internal point }
\end{array}\right\}, \\
& \Delta_{i j}=1-\Gamma_{i j} .
\end{aligned}
$$

Our computational formulas are written

$$
\begin{aligned}
& w_{i j}^{i-1 i-1}=B_{i j}^{i-1 j-1} \Gamma_{i-1 i-1}, \\
& w_{i j}^{i-1 i}=\left(B_{i j}^{i-1 i}-w_{i j}^{i-1 i-1} b_{i-1 i-1}^{i-1 j}\right) \Gamma_{i-1 i}, \\
& w_{i j}^{i-1 i}=\left(B_{i j}^{i j-1}-w_{i j}^{i-1 i-1} b_{i-1 i-1}^{i j-1}\right) \Gamma_{i j-1}, \\
& w_{i j}^{i j}=B_{i j}^{i j}-w_{i j}^{i-1 i-1} b_{i-1 i-1}^{i j}-w_{i j}^{i-1 i} b_{i-1 i}^{i j}-w_{i j}^{i j-1} b_{i j-1}^{i j} \text {, } \\
& b_{i j}^{i+1 i+1}=\frac{B_{i j}^{i+1 i+1}}{w_{i j}^{i j}} \Gamma_{i+1 i+1}, \\
& b_{i j}^{i+1 i}=\frac{B_{i j}^{i+1 i}-w_{i j}^{i j-1} b_{i j-1}^{i+1 j}}{w_{i j}^{i j}} \Gamma_{i+1 i}, \\
& b_{i j}^{i j+1}=\frac{B_{i j}^{i j+1}-w_{i j}^{i-1 i} b_{i-1 i}^{i j+1}}{w_{i j}^{i j}} \Gamma_{i j+1}, \\
& \beta_{i j}^{i-1 i+1}=w_{i j}^{i-1 i} b_{i-1 j}^{i-1 i+1} \Gamma_{i-1 j+1}, \\
& \beta_{i j}^{i+1 j-1}=w_{i j}^{i j-1} b_{i j-1}^{i+1 j-1} \Gamma_{i+1 j-1}, \\
& D_{i j}=S_{i j}-\left\{B_{i j}^{i-1 i-1} \theta_{i-1 i-1} \Delta_{i-1 i-1}+B_{i j}^{i-1 i} \theta_{i-1 i} \Delta_{i-1 i}\right. \\
& +B_{i j}^{i-1 i+1} \theta_{i-1 j+1} \Delta_{i-1 i+1}+B_{i j}^{i j-1} \theta_{i j-1} \Delta_{i j-1} \\
& +B_{i j}^{i j+1} \theta_{i j+1} \Delta_{i j+1}+B_{i j}^{i+1 i-1} \theta_{i+1 i-1} \Delta_{i+1 i-1} \\
& \left.+B_{i j}^{i+1 i} \theta_{i+1 i} \Delta_{i+1 j}+B_{i j}^{i+1 i+1} \theta_{i+1 i+1} \Delta_{i+1 i+1}\right\} \text {, }
\end{aligned}
$$

where $S_{i j}$ is a term arising from sources and the values of $\theta$ accompanying non-zero $\Delta$ are boundary values.

$$
\begin{aligned}
E_{i j}=D_{i j}-\left\{\left(B_{i j}^{i-1 i+1}-\beta_{i j}^{i-1 i+1}\right) \theta_{i-1 i+1}^{*} \Gamma_{i-1 i+1}\right. \\
\left.+\left(B_{i j}^{i+1 i-1}-\beta_{i j}^{i+1 i-1}\right) \theta_{i+1 i-1}^{*} \Gamma_{i+1 i-1}\right\},
\end{aligned}
$$

where $\theta^{*}$ denotes a guessed value.

$$
\begin{aligned}
& g_{i i}=\frac{E_{i j}-w_{i j}^{i j-1} g_{i j-1} \Gamma_{i j-1}-w_{i j}^{i-1 i} g_{i-1 j} \Gamma_{i-1 i}-w_{i j}^{i-1 i-1} g_{i-1 i-1} \Gamma_{i-1 i-1}}{w_{i j}^{i j}}, \\
& \theta_{i j}=g_{i j}-b_{i j}^{i j+1} \theta_{i j+1} \Gamma_{i j+1}-b_{i j}^{i+1 j} \theta_{i+1 i} \Gamma_{i+1 i}-b_{i j}^{i+1 i+1} \theta_{i+1 i+1} \Gamma_{i+1 i+1} .
\end{aligned}
$$

Some of the $\Gamma$ 's and $\Delta$ 's are redundant here since the computed factors would be zero anyway. It is understood that the formulas are to be applied only when $i j$ is an interior point of the region.

The calculational procedure for each iteration is as follows. We set our program 


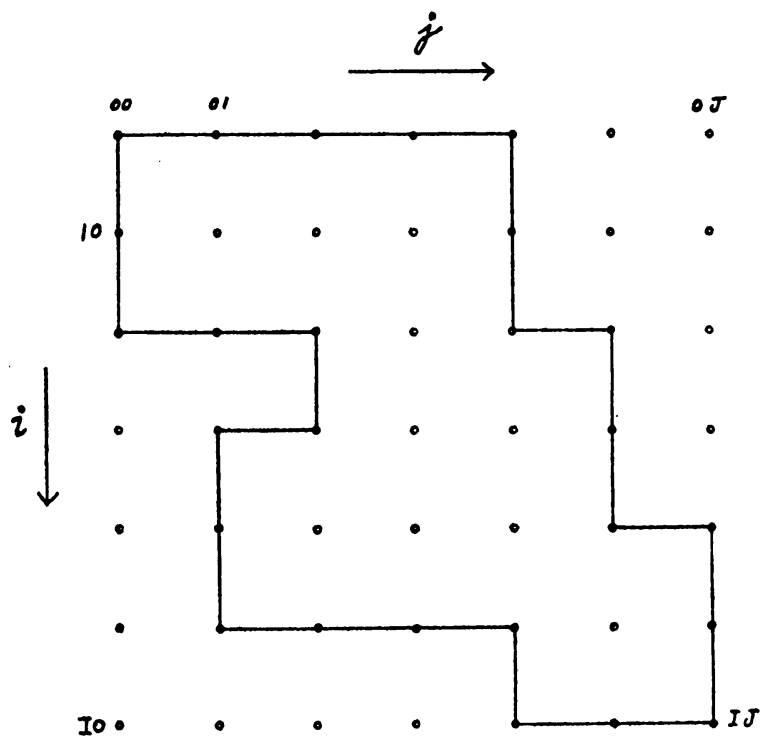

FIG. 1. A typical mesh.

to run through the whole mesh as shown in Fig. 1 from the upper left hand corner to the lower right hand corner, computing and storing the $b$ 's and $g$ 's for the entire mesh using Eqs. (27) to (38). Then we run back through the mesh from lower right to upper left computing the $\theta$ 's using Eq. (39).

4. The convergence condition for linear problems. Let us define the errors $\epsilon$ and $\epsilon^{*}$,

$$
\begin{aligned}
\theta_{\mathrm{cal} l o} & =\theta_{\mathrm{true}}+\epsilon, \\
\theta_{\mathrm{guessed}} & =\theta_{\mathrm{true}}+\epsilon^{*} .
\end{aligned}
$$

Values of $\theta_{\text {guessed }}$ are specified for the entire mesh and put in as the $\theta^{* \prime} \mathrm{s}$ of (37). Then the $\theta_{\text {calo }}$ are obtained from (39) in the course of the computation. Substituting (40) and (41) into (19) we obtain

$$
\begin{aligned}
B_{i j}^{i-1 i-1} \epsilon_{i-1 i-1}+B_{i j}^{i-1 i} & \epsilon_{i-1 i}+\beta_{i j}^{i-1 i+1} \epsilon_{i-1 i+1} \\
& +B_{i j}^{i j-1} \epsilon_{i j-1}+B_{i j}^{i j} \epsilon_{i j}+B_{i j}^{i j+1} \epsilon_{i j+1}+\beta_{i j}^{i+1 i-1} \epsilon_{i+1 i-1} \\
& +B_{i j}^{i+1 i} \epsilon_{i+1 i}+B_{i j}^{i+1 i+1} \epsilon_{i+1 i+1}=\left[\left(B_{i j}^{i-1 i+1}-\beta_{i j}^{i-1 i+1}\right) \epsilon_{i-1 i+1}^{*}\right. \\
& \left.+\left(B_{i j}^{i+1 i-1}-\beta_{i j}^{i+1 i-1}\right) \epsilon_{i+1 i-1}^{*}\right] .
\end{aligned}
$$

We can write this equation symbolically as

$$
C_{i j}^{k l} \epsilon_{k l}=D_{i j}^{k l} \epsilon_{k l}^{*} .
$$

In matrix and vector notation,

$$
C \varepsilon=D \varepsilon^{*} .
$$

The solution for $\varepsilon$ is written symbolically

$$
\varepsilon=C^{-1} D \varepsilon^{*}
$$


or

$$
\varepsilon=K \varepsilon^{*},
$$

where

$$
K=C^{-1} D
$$

Therefore we see that the convergence condition can be written in terms of the norm $\|K\|$ of $K$. It is

$$
\|K\|<1 \text {. }
$$

Thus if we can compute $\|K\|$, we can tell whether or not a given problem converges. Let us take as a definition of the norm, the following expression

$$
\|K\|=\operatorname{Max}_{\left(\mathbf{v}_{i} \mid\right.}\left[\frac{\left|K \mathbf{v}_{i}\right|}{\mathbf{v}_{i}}\right] \text {, }
$$

where the $\boldsymbol{\nabla}_{\boldsymbol{i}}$ form a complete set of linearly independent vectors in the space of the problem. The process for obtaining $K \mathbf{v}_{i}$ from $\mathbf{v}_{i}$ is identical to the process for obtaining $\varepsilon$ from $\varepsilon^{*}$ by the use of (42). Equation (42) is similar to our original problem as given by (2); however, now we already have the $\beta$ 's on the left hand side of (42) so that it automatically satisfies the consistency conditions (16) and (17). Therefore, no iterations are required here.

We thus investigate the convergence of a particular problem in the following way. First, we select the set of vectors $\boldsymbol{\nabla}_{i}$. Then using (42), we compute the vectors $K \mathbf{v}_{i}$. Then using Eq. (49) we compute the norm $\|K\|$. If the convergence condition is satisfied, we proceed to obtain the solution.

5. Numerical examples. As our linear example we will take the heat flow problem discussed by Baker and Oliphant [3]. The differential equation is

$$
\frac{\partial^{2} \theta}{\partial x^{2}}+\frac{\partial^{2} \theta}{\partial y^{2}}=\alpha^{-2} \frac{\partial \theta}{\partial t},
$$

where $\alpha$ is a constant. The left hand side can be differenced either

$$
L_{+} \theta=\frac{\theta_{i-1 i}+\theta_{i+1 i}+\theta_{i j-1}+\theta_{i i+1}-4 \theta_{i i}}{(\Delta x)^{2}}
$$

or

$$
L_{x} \theta=\frac{\theta_{i-1 i-1}+\theta_{i+1 i-1}+\theta_{i-1 i+1}-4 \theta_{i j}}{2(\Delta x)^{2}} .
$$

For our actual scheme, we take a weighted superposition of the schemes illustrated in (51) and (52). Thus,

$$
\alpha_{+} L_{+} \theta+\alpha_{x} L_{x} \theta=\alpha^{-2} \frac{\partial \theta}{\partial t}
$$

where

$$
\alpha_{+}+\alpha_{x}=1 \text {. }
$$

We now make a direct comparison with the linear problem worked by Baker and 
Oliphant. For simplicity, we set $(\Delta x)=(\Delta y)$. The time derivative is replaced by a three-level formula [4]

$$
\frac{\partial \theta}{\partial t}=\frac{3 \theta_{i i}^{n}-4 \theta_{i j}^{n-1}+\theta_{i j}^{n-2}}{2(\Delta t)} .
$$

Substituting (55) into (53), we obtain

$$
\alpha_{+} L_{+} \theta+\alpha_{x} L_{x} \theta+\beta \theta=\alpha^{-2} \frac{\left(\theta_{i j}^{n-2}-4 \theta_{i j}^{n-1}\right)}{2(\Delta t)}
$$

where

$$
\beta=-\frac{3}{2 \alpha^{2}(\Delta t)}
$$

A simple algebraic manipulation shows that the Baker-Oliphant scheme for $(\Delta x)=(\Delta y)$ can be obtained by setting

$$
\begin{aligned}
& \alpha_{+}=1-\frac{2}{\beta(\Delta x)^{2}}, \\
& \alpha_{x}=\frac{2}{\beta(\Delta x)^{2}} .
\end{aligned}
$$

We observe that (54) is satisfied by (58) and (59).

As in the particular example considered by Baker and Oliphant, we choose $\alpha^{2}(\Delta t) /(\Delta x)^{2}=1.02392228$ so that the amplitude is diminished by a factor $10^{-1 / 16}$ at each time step. For this example, letting $(\Delta x)=1 / 12$, we have

$$
\begin{aligned}
\beta & =-210.953511, \\
\alpha_{+} & =2.3652297 \\
\alpha_{x} & =-1.3652297 .
\end{aligned}
$$

We carried this out on an IBM 704. The calculation gave the same result as the BakerOliphant calculation. It was observed by them that the solution for a single time step is exact and requires no iterations. Equivalently, we observe here that the $\beta_{i j}^{i-1 i+1}$ and $\beta_{i j}^{i+1 i-1}$ of Eqs. (34) and (35) are precisely equal to the $B_{i j}^{i-1 i+1}$ and $B_{i j}^{i+1 i-1}$, respectively, so that the coefficients of $\theta_{i-1 i+1}^{*}$ and $\theta_{i+1 i-1}^{*}$ in (37) are precisely zero.

Thus, we see how in this case our method reduces to the Baker-Oliphant method as a special case. We can look at the relationship between the two methods in the following way. We obtain more generality by introducing the iterations on each time step. When we specialize to the Baker-Oliphant method we have no need for the iterations because the solution is obtained correctly in one step.

To illustrate how our method is applied to a scheme which does not permit the BakerOliphant factorization, we considered an example with weights

$$
\begin{gathered}
\alpha_{+}=2 / 3, \\
\alpha_{x}=1 / 3 .
\end{gathered}
$$

As a criterion for iteration we required that successive iterates be within one part in $10^{3}$ of each other. We found that for this example four iterations were required per time 
step. In situations in which we can obtain a very good extrapolated guess for the succeeding time step, we expect not to have an excessively large number of iterations, for example, some 5 or 6 . This expectation has been borne out in a number of calculations of the above type. However, even in differencing schemes to which the Baker-Oliphant factorization does not apply precisely, it is advisable to choose weights closely corresponding to their method in order to reduce the number of iterations as much as possible. In calculating linear examples, the program runs at about 13 milliseconds per cycle point.

For our non-linear example we considered the same example as that used by Baker and Oliphant, namely radiation flow in a material medium. The equation is

$$
\nabla^{2}\left(\theta^{4}\right)=16 K \frac{\partial \theta}{\partial t} .
$$

With the present method, we need not make the change of variable required in the BakerOliphant scheme, but we can simply linearize the second partial derivative term. We use a linearization which is similar to that used by Bruce, Peaceman, Rachford, and Rice [2] in their one-dimensional examples. The resulting difference scheme can be written:

$$
\begin{aligned}
\alpha_{x}\left(B_{i j}^{i-1 i-1} \theta_{i-1 i-1}+\right. & \left.B_{i j}^{i-1 i+1} \theta_{i-1 i+1}+B_{i j}^{i+1 i-1} \theta_{i+1 i-1}+B_{i j}^{i+1 i+1} \theta_{i+1 i+1}\right) \\
& +\alpha_{+}\left(B_{i j}^{i-1 i} \theta_{i-1 i}+B_{i j}^{i+1 i} \theta_{i+1 i}+B_{i j}^{i i-1} \theta_{i j-1}+B_{i j}^{i j+1} \theta_{i j+1}\right) \\
& -\left[\left(\alpha_{x} B_{i j}^{i j}(x)+\alpha_{+} B_{i j}^{i j}(+)+\beta\right)\right] \theta_{i j}=16 K \frac{\left(\theta_{i j}^{n-2}-4 \theta_{i j}^{n-1}\right)}{2(\Delta t)}
\end{aligned}
$$

where

$$
B_{i j}^{m n}=f\left(\theta_{i j}^{*}, \theta_{m n}^{*}\right) \equiv\left(\theta_{i j}^{*}\right)^{3}+\left(\theta_{i j}^{*}\right)\left(\theta_{m n}^{*}\right)+\left(\theta_{i j}^{*}\right)\left(\theta_{m n}^{*}\right)^{2}+\left(\theta_{m n}^{*}\right)^{3},
$$

except for $i=m, j=n$,

$$
\begin{array}{r}
B_{i j}^{i j}(x)=f\left(\theta_{i j}^{*}, \theta_{i-1 i-1}^{*}\right)+f\left(\theta_{i j}^{*}, \theta_{i-1 i+1}^{*}\right)+f\left(\theta_{i j}^{*}, \theta_{i+1 i-1}^{*}\right)+f\left(\theta_{i j}^{*}, \theta_{i+1 i+1}^{*}\right), \\
B_{i j}^{i j}(+)=f\left(\theta_{i j}^{*}, \theta_{i-1 i}^{*}\right)+f\left(\theta_{i j}^{*}, \theta_{i+1 j}^{*}\right)+f\left(\theta_{i j}^{*}, \theta_{i j-1}^{*}\right)+f\left(\theta_{i j}^{*}, \theta_{i+1}^{*}\right),
\end{array}
$$

and

$$
\beta=-\frac{24 K}{(\Delta t)} .
$$

In the non-linear case we see that the $\theta^{* \prime}$ s occur on the left-hand side as well as the right-hand side. The procedure to be followed in accordance with the analysis of Sec. 4 would be to put a guess $\theta^{* *}$ into the coefficients given by Eqs. (67) to (69) and iterate $\theta^{*}$ in Eq. (37) down as in a linear equation, and then to make another guess $\theta^{* *}$ and so on. In practice we find it much better to guess $\theta^{*}$ for both (67) to (69) and (37) at the same time. The latter procedure was followed in the example discussed below.

For our sample calculation we picked $\beta \Delta x^{2}=\beta \Delta y^{2}=-1.5$ and used $11 \times 11$ interior mesh points. For initial conditions we chose

$$
\theta(x, y)=1-\sin \left(\frac{2 \pi x}{L}\right) \sin \left(\frac{2 \pi y}{L}\right)
$$


and we maintained the boundaries at unit temperature. This is the identical example chosen by Baker and Oliphant. Initially some 6 or 8 iterations were required per time step, but after 16 time steps, only about 2 iterations per time step were required for three-place accuracy. We made the initial guess at each time step by a linear extrapolation from the previous two times. The program runs at about 47 milliseconds per cyclepoint.

As with the Baker-Oliphant method, care should be exercised to prevent the guessing of a negative temperature, as the occurrence of temperatures of different signs produces an instability which causes the solution to diverge.

As was observed in the above calculations, the convergence of the iterations was rather good. We can discuss why this should be so in the linear case on the basis of the analysis of Sec. 4. We computed the norm of (49) for a number of sample matrices. It was found that the convergence is best for the heaviest weighting of the diagonal element of $B_{i j}^{k 1}$. On the left-hand side of Eq. (56), the first two terms weight the diagonal and off-diagonal terms equally. In steady state problems, these are the only terms present and for such problems we get fairly good convergence. For the time-dependent problems, the third term adds on in such a way as to increase the weighting of the diagonal elements and thus to enhance the convergence greatly. Also, in time-dependent problems, taking extrapolated guesses helps considerably.

\section{REFERENCES}

1. J. Douglas, Jr., and D. W. Peaceman, Numerical solutions of two-dimensional heat-flow problems, A. I. Ch. E. Journal 1, 505-512 (1955)

2. G. H. Bruce, D. W. Peaceman, H. H. Rachford, Jr., and J. D. Rice, Calculation of unsteady-state gas flow through porous media, Trans. Am. Inst. Mining Met. Engrs. 198, 79-92 (1953)

3. G. A. Baker, Jr., and T. A. Oliphant, An implicit, numerical method for solving the two-dimensional heat equation, Quart. Appl. Math. 17, 361-373 (1960)

4. R. D. Richtmyer, Difference methods for initial-value problems, Interscience Publishers, Inc., New York, 1957 